\title{
Acute Kidney Injury Masquerading as Acute Pancreatitis - Role of Serum Amylase and Lipase in Patients with Blunt Abdominal Trauma
}

\author{
Sharad Sonawane ${ }^{1}$, Parth Godhiwala ${ }^{2}$, Srinivas Naik ${ }^{3}$, Iftekhar Ansari ${ }^{4}$, Sunil Kumar ${ }^{5}$
}

\begin{abstract}
${ }^{1}$ Department of Medicine, Jawaharlal Nehru Medical College, Datta Meghe Institute of Medical Sciences (Deemed to Be University), Wardha, Maharashtra, India. ${ }^{2}$ Department of Medicine, Jawaharlal Nehru Medical College, Datta Meghe Institute of Medical Sciences (Deemed to Be University), Wardha, Maharashtra, India. ${ }^{3}$ Department of Medicine, Jawaharlal Nehru Medical College, Datta Meghe Institute of Medical Sciences (Deemed to Be University), Wardha, Maharashtra, India. ${ }^{4}$ Department of Medicine, Jawaharlal Nehru Medical College, Datta Meghe Institute of Medical Sciences (Deemed to Be University), Wardha, Maharashtra, India. ${ }^{5}$ Department of Medicine, Jawaharlal Nehru Medical College, Datta Meghe Institute of Medical Sciences (Deemed to Be University), Wardha, Maharashtra, India.
\end{abstract}

\section{INTRODUCTION}

Blunt trauma to abdomen is one of the common causes for morbidity and mortality. In blunt abdominal trauma, there may be trauma to the pancreas, tear of bowel and hollow viscous, which can result in elevated levels of serum amylase and lipase. Serum amylase and mainly serum lipase are diagnostic markers for pancreatitis according to some literature. We report a young male, who presented with complaints of acute abdominal pain, constipation and vomiting after blunt abdominal trauma with elevated serum amylase and lipase levels with normal pancreas.

Initial assessment of intra-abdominal pathology due to blunt abdominal trauma is often challenging as many injuries may not manifest during the treatment period. The manifestations may vary from being simply asymptomatic to life threatening injuries due to unavailability of accurate information about the nature of trauma. Hence, accurate diagnosis becomes very important in the management. Injury to the pancreas is not common and can result from direct penetration or through the transmission of blunt force to the retroperitoneum. Some studies report an incidence of $0.2 \%$ cases for blunt trauma up to $1.1 \%$ cases for penetrating trauma. ${ }^{1}$ The role of ultrasonography (USG) and Computed Tomography (CT) in management of Blunt abdominal trauma is well established. But the role of laboratory tests in the evaluation of these patients is controversial. ${ }^{2}$ In cases of Blunt trauma to abdomen, lot of causes found to cause serum amylase and serum lipase levels tend to raise. Some of these causes can be pancreatic, rupture of hollow viscus or bowel, facial and brain injury. ${ }^{3}$ It has also been found that reduced clearance of lipase can be caused by renal impairment. Thus non-pathological raise in level of pancreatic enzymes, and other miscellaneous causes such as diabetes mellitus, drugs and infections shall be included as alternative pancreatic diagnoses. ${ }^{3}$

\section{PRESENTATION OF CASE}

A 20 year old young male patient presented to the emergency department with alleged history of blunt abdominal trauma while working in a cotton factory followed by complaints of acute abdominal pain and constipation since 3 days and history of vomiting ( 3 episodes per day) since 2 days.
Corresponding Author: Dr. Parth Godhiwala. Department of Medicine, Jawaharlal Nehru Medical College, Datta Meghe Institute of Medical Sciences (Deemed to Be University), Wardha, Maharashtra, India.

E-mail: persie_parth@yahoo.in

DOI: $10.14260 / \mathrm{jemds} / 2020 / 601$

How to Cite This Article:

Sonawane S, Godhiwala P, Naik S, et al. Acute kidney injury masquerading as acute pancreatitis - role of serum amylase and lipase in patients with blunt abdominal trauma. J Evolution Med Dent Sci 2020;9(37):2767-2769, $10.14260 / \mathrm{jemds} / 2020 / 601$

Submission 12-06-2020,

Peer Review 05-08-2020,

Acceptance 13-08-2020,

Published 14-09-2020.

Copyright (C) 2020 JEMDS. This is an open access article distributed under Creative Commons Attribution License [Attribution 4.0 International (CC BY 4.0)] 
He also complained of decrease in urine output and burning micturition with no history of blood in urine and stools, chest pain, palpitations, shortness of breath, orthopnea, paroxysmal nocturnal dyspnoea. Patient had no past history of systemic hypertension, diabetes. He had no history of smoking and alcohol intake. On examination, he was ectomorphic, afebrile, pulse rate of $78 \mathrm{bpm}$, regular, his blood pressure was $130 / 80 \mathrm{~mm}$ hg. He had absent bilateral pitting oedema feet and on abdominal examination, there was diffuse tenderness in right quadrants of abdomen. There was guarding and rigidity of the abdomen was present.

Laboratory investigations revealed following values.

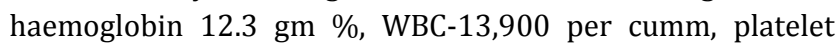
count 1.05 lakh per cumm, urea $69 \mathrm{mg} / \mathrm{dL}$, creatinine $4.2 \mathrm{mg}$ / dL, potassium $5.9 \mathrm{mmol} / \mathrm{L}$, sodium $145 \mathrm{mmol}$ / L, serum lactate dehydrogenase (LDH) $1290 \mathrm{U} / \mathrm{L}$, liver function test was normal. In view of persistent acute pain in abdomen, his serum amylase and lipase were measured and was made to undergo NCCT.

His NCCT abdomen which was suggestive of hepatomegaly and right renal calculus with perinephric collection on right side and haemoperitoneum possibility of right renal injury [Figure 1] with right sided pleural effusion, pancreas was normal. Patient's ultrasonography was suggestive of raised echotexture of bilateral kidneys with lost corticomedullary differentiation, bilateral pleural effusion right more than left, grade 3 renal parenchymal disease, pancreas normal size, shape and echotexture. His serum amylase and serum lipase done on 1, 4 and 7 days [Table 1] on $1^{\text {st }}$ day it came out to be $6360 \mathrm{U} / \mathrm{L}$ and $16945 \mathrm{U} / \mathrm{L}$ respectively. On day 4 serum amylase and lipase were $638 \mathrm{U} / \mathrm{L}$ and $1015 \mathrm{U} / \mathrm{L}$ and on 7th day serum amylase and lipase were $276 \mathrm{U} / \mathrm{L}$ and $781 \mathrm{U} / \mathrm{L}$ respectively. His creatine kinase (CK) was 366 U / L. ECG was normal. Patient also had decrease urine output thus he was managed with plenty of fluids and other supportive medications.

\begin{tabular}{|cccccc|}
\hline Days & $\begin{array}{c}\text { Hb } \\
\text { (gm \%) }\end{array}$ & $\begin{array}{c}\text { Serum Amylase Serum Lipase } \\
\text { (U / L) }\end{array}$ & $\begin{array}{c}\text { Serum } \\
\text { Creatinine } \\
\text { ( } / \text { L) }\end{array}$ & $\begin{array}{c}\text { Serum } \\
\text { Urea (mg) } \\
\text { / dL) }\end{array}$ \\
\hline 1 & 12.3 & 6360 & 16945 & 4.2 & 103 \\
4 & 7.7 & 638 & 1015 & 2.8 & 69 \\
7 & 7.1 & 276 & 781 & 1.3 & 30 \\
\hline \multicolumn{5}{|r}{ Table 1. Decreasing Trends of Serum Amylase and Serum Lipase } \\
Levels after Resolving Acute Kidney Injury
\end{tabular}

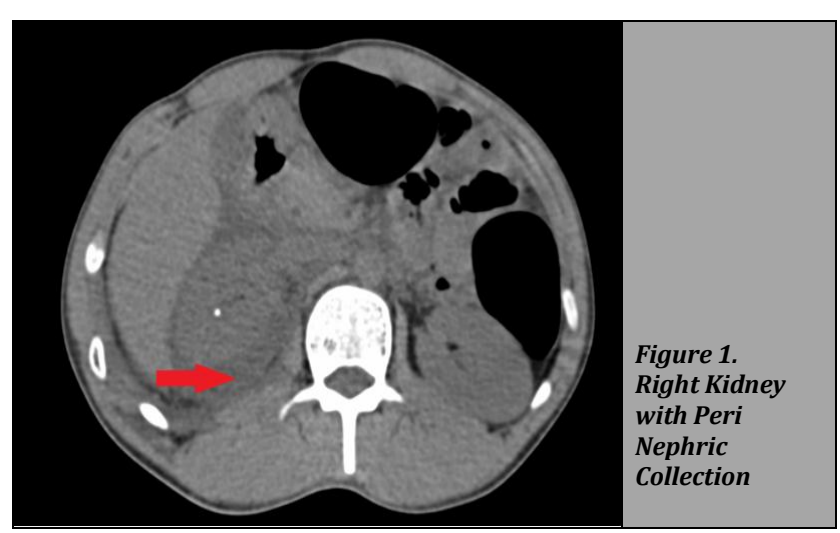

Injury to pancreas are relatively unusual but, however represent serious problem because of high morbidity and mortality rates that go with them, with morbidity rates ranging between $45 \%$ and $60 \%$, and mortality rates ranging between $23.4 \%$ and $30.2 \%{ }^{4}$ Pancreatic injury is a common problem for diagnosis following blunt abdominal trauma as symptoms are often insidious in onset and enzyme markers (amylase and lipase) are known not to correlate with severity of injury. Recent studies suggestive of 6 hours after trauma serum amylase and lipase levels raised, and can be used as reliable indicator of injury to the pancreas, however non diagnostic within 6 hours or less after trauma. ${ }^{5}$ In the event that the abdominal assessment at first yields ambiguous or questionable outcomes in a haemodynamically steady patient with hyperamylasaemia, a complexity improved abdominal CT output ought to be finished. We had ruled out any obstructive pathology in abdomen by abdominal ultrasonography and by CT. ${ }^{6}$

Building up the nearness of pancreatic injury is an extensive test, as no single imaging methodology has been found to exceptionally compelling. In this way, for ahead of schedule and convenient conclusion of pancreatic injury, a high file of doubt must be kept up and pancreas can likewise be ordinary in an extent of wounds. ${ }^{4}$ In clinical renal failure Serum lipase can increase through the progression of renal dysfunction. ${ }^{7}$ These enzymes may also be elevated due to defect in tubular reabsorption of amylase and lipase. ${ }^{8}$ Reduced clearance of lipase from the circulation can occur with renal impairment or macro lipase formation. There are many potential causes other than pancreatitis for the significant elevation of lipase levels. Lipase may also be elevated as a result of other intra-abdominal pathologies arising from the stomach, bowel and hepatobiliary tract, neoplastic disease. ${ }^{3}$

In our case, laboratory tests with raised serum amylase and lipase and NCCT scan of the abdomen, suggested normal pancreas, hepatomegaly and right renal calculus with perinephric collection on right side and haemoperitoneum possibility of right renal injury with right sided pleural effusion. Thus pancreatitis was rule out and patient was treated as a case of acute kidney injury. ${ }^{9}$

\section{CONCLUSIONS}

Injury to pancreas is a common diagnostic dilemma following blunt abdominal trauma as symptoms are often insidious in onset and enzyme markers (amylase and lipase) are elevated. But serum lipase can also increase due to clinical renal failure and may tend to increase through the progression of renal dysfunction. Thus any patient with blunt trauma, raised enzymes and normal pancreas must be evaluated for acute kidney injury to prevent serious complications.

Financial or Other Competing Interests: None.

\section{REFERENCES}

[1] Akhrass R, Yaffe MB, Brandt CP, et al. Pancreatic trauma: a ten-year multi-institutional experience. Am Surg 1997;63(7):598-604.

[2] Debi U, Kaur R, Prasad KK, et al. Pancreatic trauma: a concise review. World J Gastroenterol 2013;19(47):900311. 
[3] Hameed AM, Lam VWT, Pleass HC. Significant elevations of serum lipase not caused by pancreatitis: a systematic review. HPB (Oxford) 2015;17(2):99-112.

[4] Hasanovic J, Agic M, Rifatbegovic Z, et al. Pancreatic injury in blunt abdominal trauma. Med Arch 2015;69(2):130-2.

[5] Mahajan A, Kadavigere R, Sripathi S, et al. Utility of serum pancreatic enzyme levels in diagnosing blunt trauma to the pancreas: a prospective study with systematic review. Injury 2014;45(9):1384-93.

[6] Kumar S, Jain AP, Pandit AK. Acute pancreatitis: rare complication of chicken pox in an immunocompetent host. Saudi J Gastroenterol 2007;13(3):138-140.
[7] Zhu Y, Dong J, Wang P, et al. Serum lipase as clinical laboratory index for chronic renal failure diagnosis. Clin Lab 2016;62(7):1265-70.

[8] Marin RS, Meredith JW. Management of acute trauma. In: Townsend C, Beauchamp D, Evers M, et al, eds. Sabiston Textbook of surgery: the biological basis of modern surgical practice. Philadelphia: Elsevier/Saunders 2012:430-70.

[9] Kumar S, Diwan SK, Dekate M. Chronic pancreatitis presenting as recurrent pleural effusion. Online J Health Allied Scs 2013;12(2):22. 